
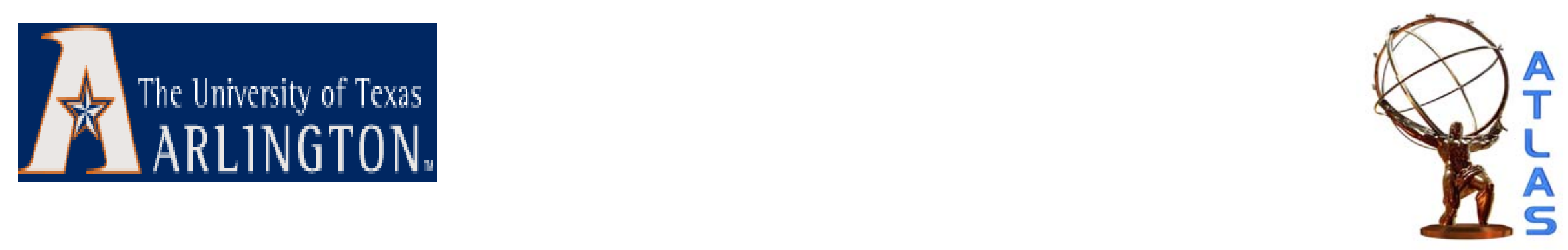

\title{
Exclusive Measurements for SUSY Events with the ATLAS Detector at the LHC
}

17th International Conference on Supersymmetry and the Unification of Fundamental Interactions, SUSY'09 Boston, June 5-19, 2009

Nurcan Ozturk University of Texas at Arlington On behalf of the ATLAS collaboration 


\section{Outline}

- Introduction

- SUSY Measurements

- mSUGRA Benchmark Points

- Mass Measurements

- Dilepton endpoint

- Leptons+jets endpoints

- Ditau endpoint

- Right handed squark mass

- Light stop mass

- From Endpoints to SUSY Masses

- From Endpoints to mSUGRA Parameters

- Conclusions 


\section{Introduction}

- Supersymmetry (SUSY) can be discovered by the ATLAS experiment at the LHC during the initial data taking ( first year), if SUSY particles have masses at sub-TeV range.

- A strategy for SUSY discovery by ATLAS has been outlined in the following talks:

- "Prospects for SUSY discovery based on inclusive searches with the ATLAS detector at the LHC", by Xavier Portell.

- "Multi-lepton SUSY searches with the ATLAS detector", by Katarina Pajchel.

- "Reconstruction of tau leptons and prospects for SUSY in ATLAS", by Carolin Zendler.

- This talk: Recent work performed in ATLAS on the techniques used to reconstruct the decays of SUSY particles, based on the selection of final-state exclusive decay chains. Concentrate on strategies to be applied to the first $\mathrm{fb}^{-1}$ of LHC data.

- Reference - CERN report: "Expected Performance of the ATLAS Experiment Detector, Trigger and Physics" by the ATLAS Collaboration, CERN-OPEN-2008-020, arXiv:0901.0512. 


\section{SUSY Measurements}

- As soon as a SUSY discovery (if SUSY exists in nature) is made by inclusive searches $\rightarrow$ make measurements to confirm that it is SUSY and of which type:

- describe the model; open decay channels, masses, branching ratios

- obtain the underlying model parameters

- measure the spin of new particles (not in this talk)

- A complete coverage of all allowed SUSY models is impossible $\rightarrow$ limit the study to mSUGRA models. Develop measurement techniques and fit methods.

- In mSUGRA with R-parity conservation:

- SUSY particles are pair produced

- Long decay chains, large mass differences between SUSY particles

- Lightest Supersymmetric Particle (LSP) is undetected

- The mass measurement strategy is to exploit kinematics of long decay chains.

- The first decay chain likely to be reconstructed:

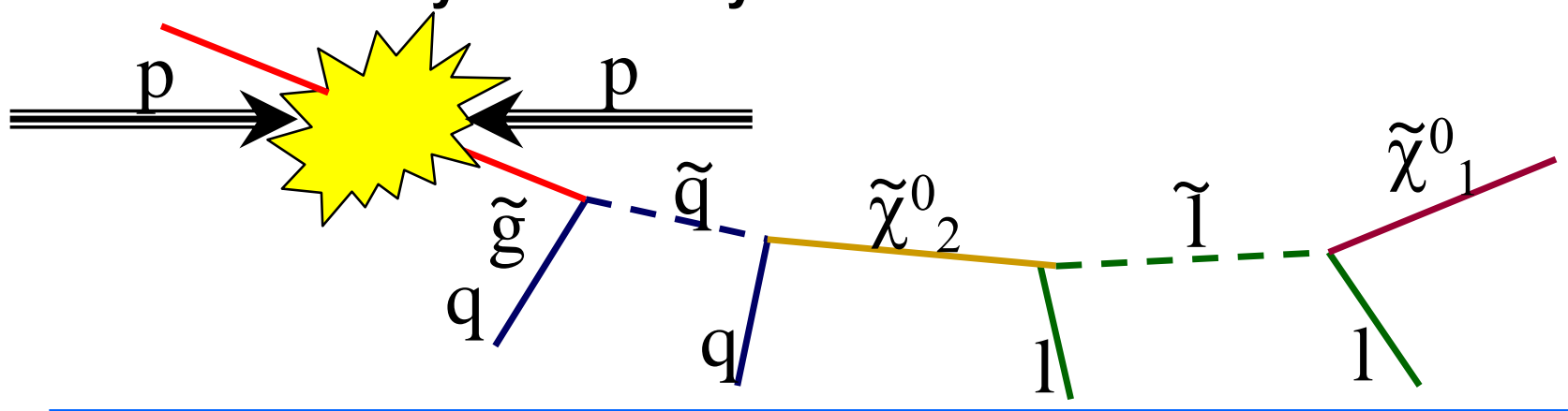

Consider mass hierarchy:

$$
\tilde{g}>\tilde{q}>\tilde{\chi}_{2}^{0}>\tilde{l}>\tilde{\chi}_{1}^{0}
$$




\section{mSUGRA Benchmark Points}

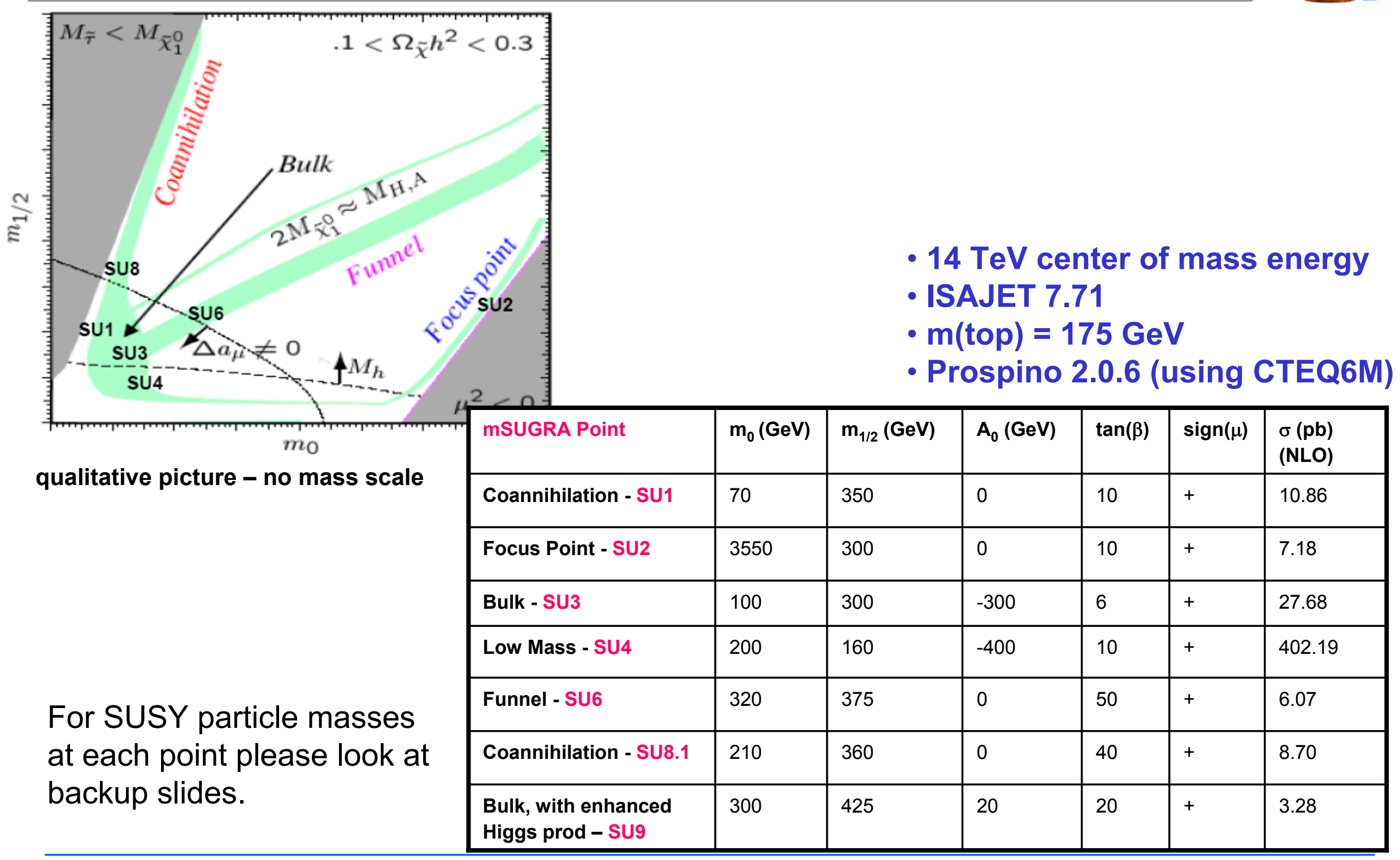




\section{Mass Measurements}

- LSP's are undetected $\rightarrow$ measure kinematic endpoints in invariant mass of visible particles, $m_{I I}, m_{I l q}, m_{l q}$, etc. rather than mass peaks.

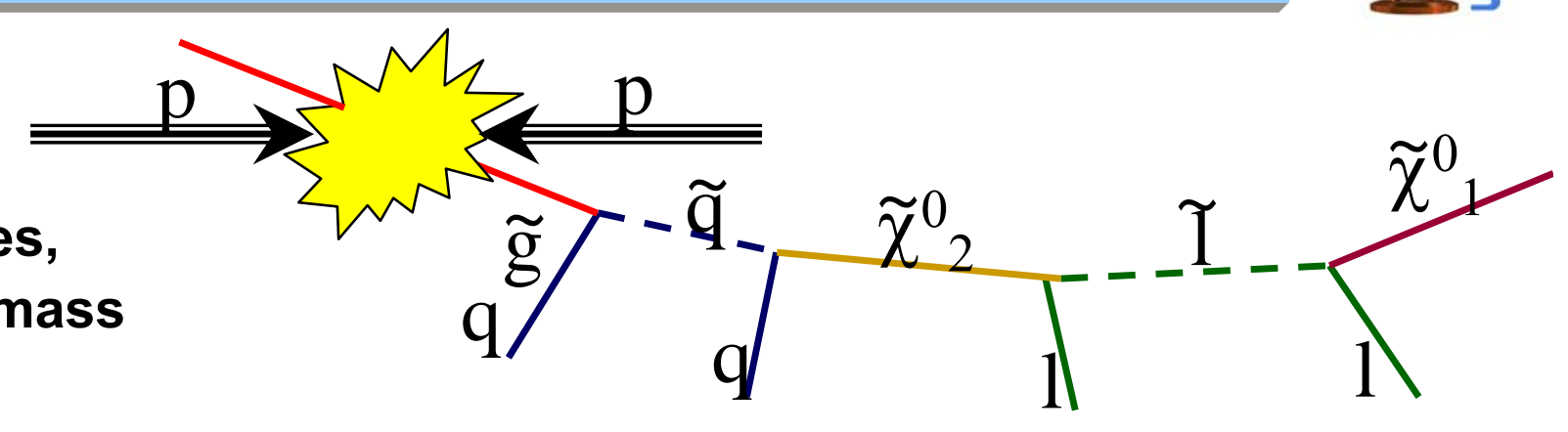

- Kinematic endpoint formulae $\rightarrow$ Allanach et al., JHEP 0009 (2000) 004, Gjelsten et al., JHEP 0506 (2005) 015.

- If sufficiently long decay chains can be isolated and enough endpoints are measured $\rightarrow$ the masses of the individual particles can be obtained.

-What can be done with $\leq 1 \mathrm{fb}^{-1}$ :

Measurements: $\mathbf{m}_{\| l}, \mathbf{m}_{\| \mathbf{l q}}, \mathbf{m}_{\| \mathbf{l q}}^{\text {thres, }}$ $m_{\text {lq }}^{\text {low }}, m_{\text {lq }}^{\text {high }}, m_{\mathrm{TT}}, m_{\mathrm{T} 2}\left(\tilde{q}_{\mathrm{R}}\right), \mathrm{m}_{\mathrm{tb}}$ Constrains (or determines) mass of:

$$
\tilde{\chi}_{1}^{0}, \tilde{\chi}_{2}^{0}, \tilde{l}_{R}, \tilde{q}_{L}, \tilde{q}_{R}, \tilde{\tau}_{1}, \tilde{t}_{1}
$$

Advantage of this decay chain:

- large signal to background ratio.

- technique, known as flavor subtraction, removes both SUSY combinatorial and Standard Model background.

Mass determination is modelindependent, but relies on interpretation of the decay chain. 


\section{Kinematic Endpoints}

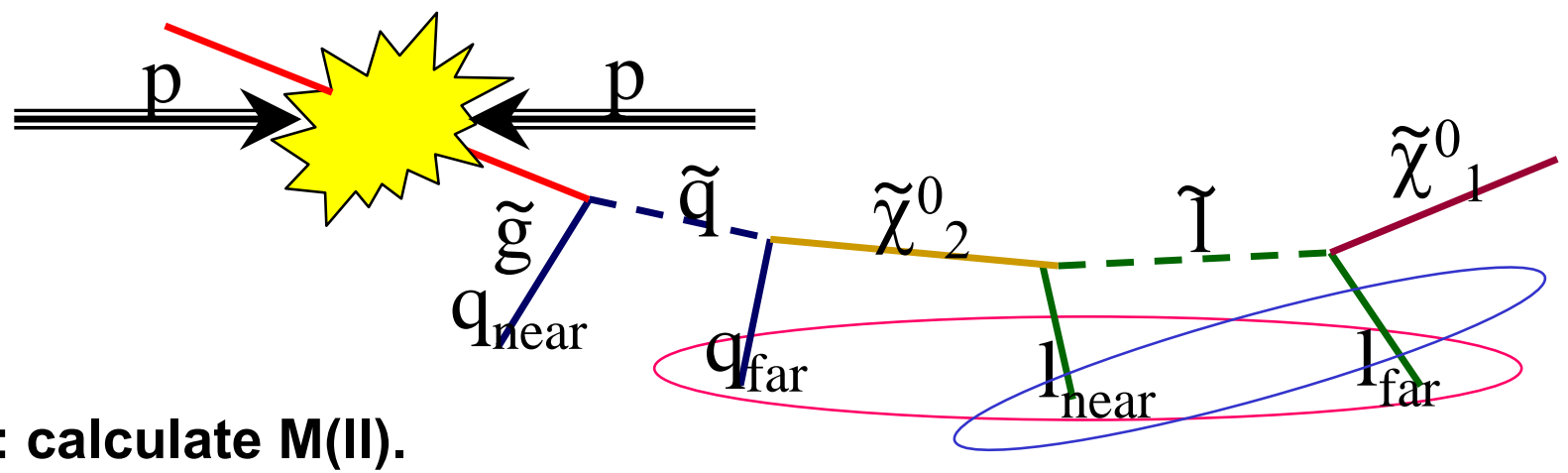

- $m_{\|}{ }^{\text {edge }}:$ calculate $M(I I)$.

- Combine the jet with leptons: Not possible to identify the quark from the squark decay $\rightarrow$ assume it generates one of the two highest $p_{T}$ jets in the event. Then:

- $m_{\| q}$ edge : calculate $M\left(\| q_{1}\right), M\left(I l q_{2}\right)$, choose the smallest.

- $m_{\| q}$ thres : calculate $M\left(I l q_{1}\right), M\left(I l q_{2}\right)$, choose the largest.

- $\mathrm{m}_{\mathrm{lq}}$ low and $\mathrm{m}_{\mathrm{lq}}$ high edges: not possible to distinguish the near lepton from the far lepton $\rightarrow$ define masses which are observable (use the jet selected for $\mathbf{m}_{\text {llq }}{ }^{\text {edge }}$ ):

$M(l q)^{\text {low }}=\min \left(M_{l^{+} q}, M_{l^{-} q}\right) \equiv \min \left(M_{l_{\text {nea }} q}, M_{l_{\text {far } q}}\right) \quad$ and $\quad M(l q)^{\text {high }}=\max \left(M_{l^{+} q}, M_{l^{-} q}\right) \equiv \max \left(M_{l_{\text {nea } q},}, M_{l_{f_{\text {ar }} q}}\right)$

- Total 5 constraints on 4 unknown SUSY masses $\rightarrow$ solvable. 


\section{Dilepton Endpoint}

- Plot invariant mass of dileptons after flavor subtraction and efficiency correction applied:

$$
\frac{M\left(e^{+} e^{-}\right)}{\beta}+\beta M\left(\mu^{+} \mu^{-}\right)-M\left(e^{ \pm} \mu^{\mp}\right)
$$

beta=ratio of electron and muon reconstruction efficiencies $\mathbf{0} .86$

- For object/particle identification and event selection please look at backup slides.

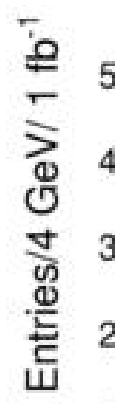
SU3 point @ $1 \mathrm{fb}^{-1}$

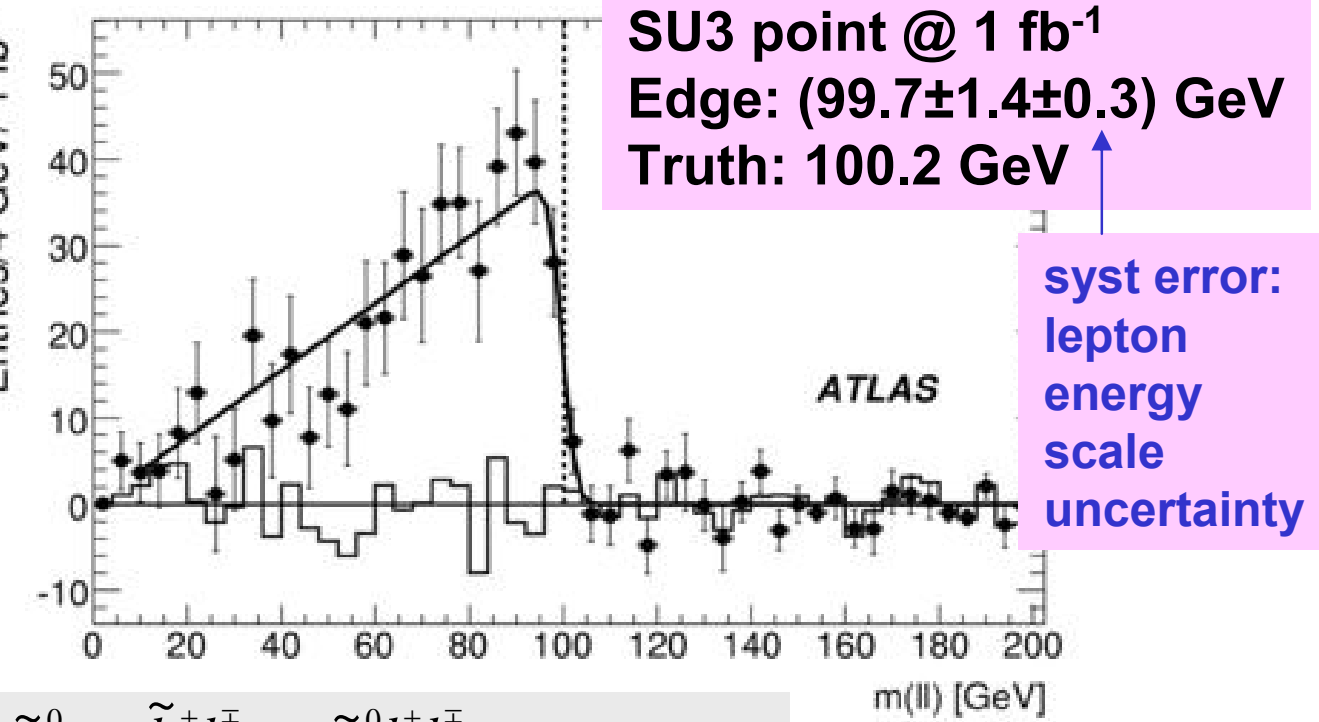

$\tilde{\chi}_{2}^{0} \rightarrow \tilde{l}^{ \pm} l^{\mp} \rightarrow \tilde{\chi}_{1}^{0} I^{ \pm} l^{\mp}$

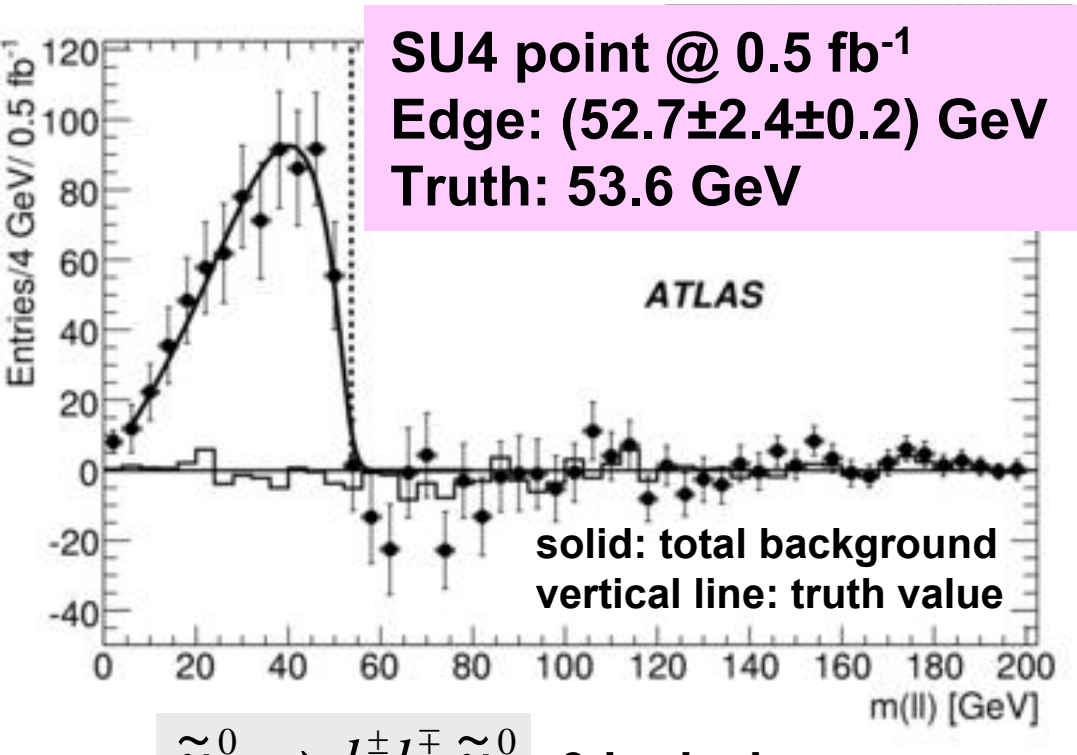

$M_{l l}^{\max }=\sqrt{\frac{\left(M_{\tilde{\chi}_{2}^{0}}^{2}-M_{\tilde{l}}^{2}\right)\left(M_{\tilde{l}}^{2}-M_{\tilde{\chi}_{1}^{0}}^{2}\right)}{M_{\tilde{l}}^{2}}}$

Fit function: triangular function smeared with a Gaussian.

Edge can be measured with a precision of a few percent already. 


\section{Leptons+jets Endpoints}
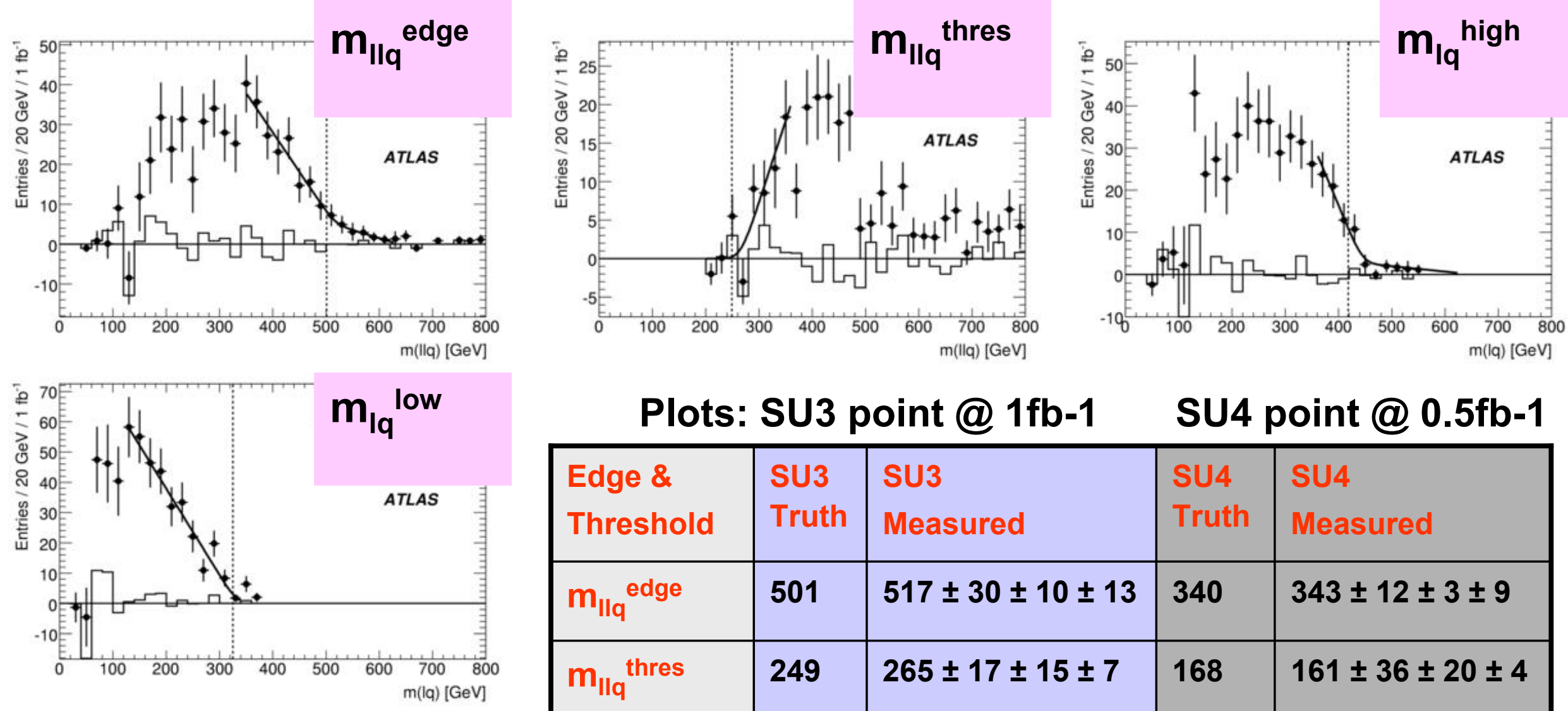

solid: total background vertical line: truth value

Very good consistency with Truth values.

\section{Plots: SU3 point @ 1fb-1 SU4 point @ 0.5fb-1}

\begin{tabular}{|l|l|l|l|l|}
\hline $\begin{array}{l}\text { Edge \& } \\
\text { Threshold }\end{array}$ & $\begin{array}{l}\text { SU3 } \\
\text { Truth }\end{array}$ & $\begin{array}{l}\text { SU3 } \\
\text { Measured }\end{array}$ & $\begin{array}{l}\text { SU4 } \\
\text { Truth }\end{array}$ & $\begin{array}{l}\text { SU4 } \\
\text { Measured }\end{array}$ \\
\hline $\mathrm{m}_{\text {Ilq }}$ edge & 501 & $517 \pm 30 \pm 10 \pm 13$ & 340 & $343 \pm 12 \pm 3 \pm 9$ \\
\hline $\mathrm{m}_{\text {Ilq }}^{\text {thres }}$ & 249 & $265 \pm 17 \pm 15 \pm 7$ & 168 & $161 \pm 36 \pm 20 \pm 4$ \\
\hline$m_{\text {Iq }}^{\text {low }}$ & 325 & $333 \pm 6 \pm 6 \pm 8$ & 240 & $201 \pm 9 \pm 3 \pm 5$ \\
\hline$m_{\text {Iq }}^{\text {high }}$ & 418 & $445 \pm 11 \pm 11 \pm 11$ & 340 & $320 \pm 8 \pm 3 \pm 8$ \\
\hline
\end{tabular}

Measured $=$ fit \pm stat \pm (lepton energy scale uncertainty) \pm (jet energy scale uncertainty) 


\section{Ditau Endpoint}

$\tilde{\chi}_{2}^{0} \rightarrow \tilde{\tau}_{1}^{ \pm} \tau^{\mp} \rightarrow \tilde{\chi}_{1}^{0} \tau^{ \pm} \tau^{\mp}$

- In SU1 and SU3 large BR for decays into tau than for decays into e/mu.

- Tau polarization carries interesting info $\rightarrow$ it can vary significantly between different SUSY models.

- Focus on hadronic tau decays.

- Neutrino energy not measured, no sharp edge.

- Strategy: fit trailing edge of spectrum with suitable function:

$$
f(x)=\frac{p_{o}}{x} \cdot \exp \left[-\frac{1}{2 p_{2}{ }^{2}}\left(\ln (x)-p_{1}\right)^{2}\right]
$$

- Endpoint is derived from the inflection point $\mathrm{m}_{\mathrm{IP}}$ of $\mathbf{f}(\mathbf{x})$ :

$$
m_{I P}=\exp \left[-\frac{1}{2} p_{2}{ }^{2}\left(3+\sqrt{\left(1-\frac{4}{p_{2}{ }^{2}}\right)}\right)+p_{1}\right]
$$

- True endpoint $m_{E P}$ is related to $m_{I P}$.

- Edge: (102 $\left.\pm 17^{\text {stat }} \pm 5.5^{\text {syst }} \pm 7^{\text {pol }}\right) \mathrm{GeV}$

- Truth: $98.3 \mathrm{GeV}$ fitting procedure polarization effects
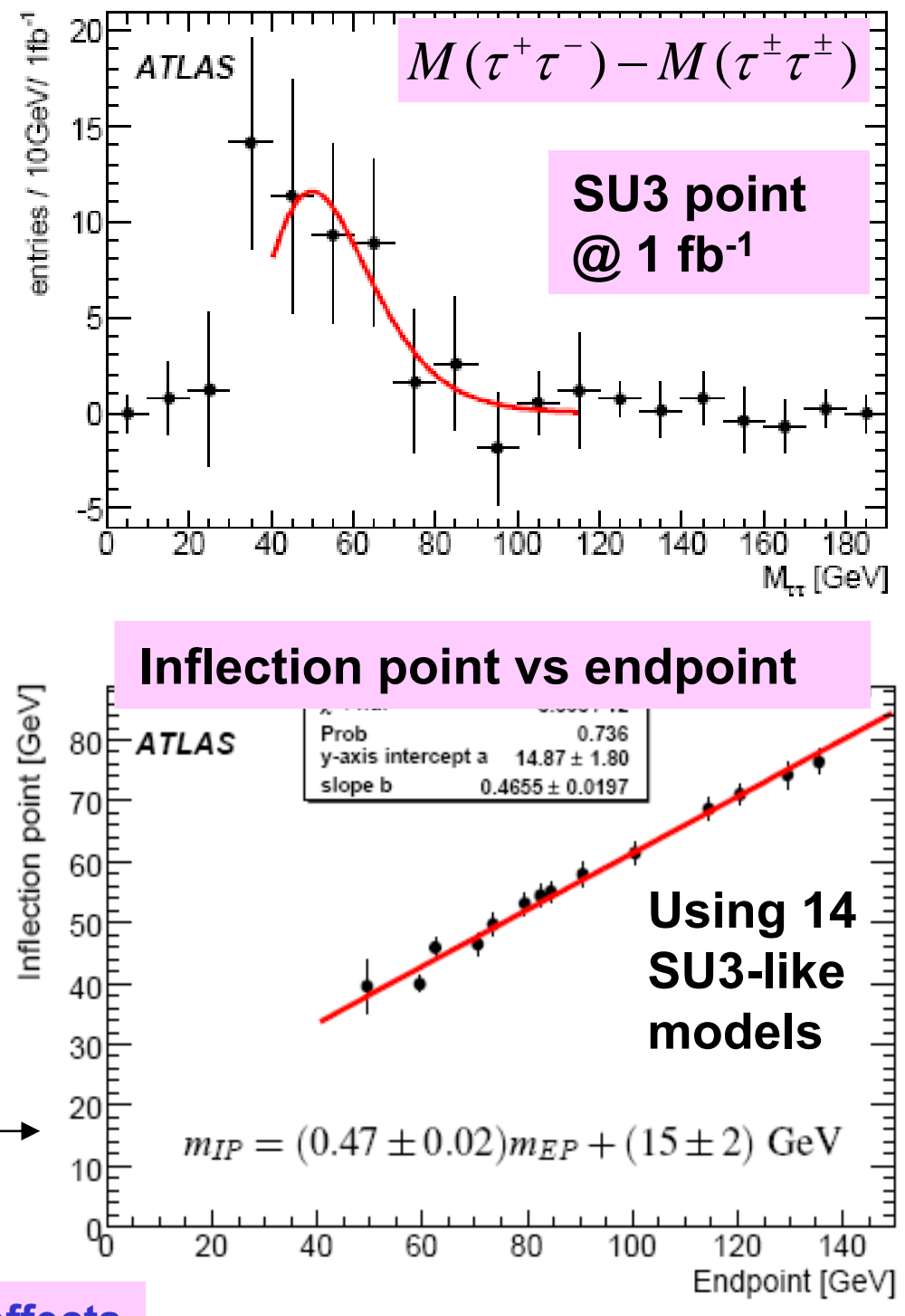


\section{Right Handed Squark Mass}

- In mSUGRA large BR for $\tilde{q}_{R} \rightarrow \tilde{\chi}_{1}^{0} q$. Signal is two hard jets plus large $E_{T}^{\text {miss. }}$.

- Calculate stransverse mass ( $m_{T 2}$ variable) of two hard jets w.r.t $E_{T}{ }^{\text {miss }} \rightarrow$ endpoint gives right-handed squark mass:

$$
m_{T 2}^{2}\left(\mathbf{p}_{T}^{\alpha}, \mathbf{p}_{T}^{\beta}, \mathbf{p}_{T}^{\text {miss }}, m_{\alpha}, m_{\beta}, m_{\chi}\right) \equiv \min _{\mathbf{q}_{T}^{(1)}+\mathbf{q}_{T}^{(2)}=\mathbf{p}_{T}^{\text {miss }}}\left[\max \left\{M_{T}^{2}\left(\mathbf{p}_{T}^{\alpha}, \mathbf{q}_{T}^{(1)} ; m_{\alpha}, m_{\chi}\right), M_{T}^{2}\left(\mathbf{p}_{T}^{\beta}, \mathbf{q}_{T}^{(2)} ; m_{\beta}, m_{\chi}\right)\right\}\right]
$$

by assuming $m_{\tilde{\chi}_{1}^{0}}$ is known from previous measurements (dilepton/leptons+jets endpoints). Use its true value below.

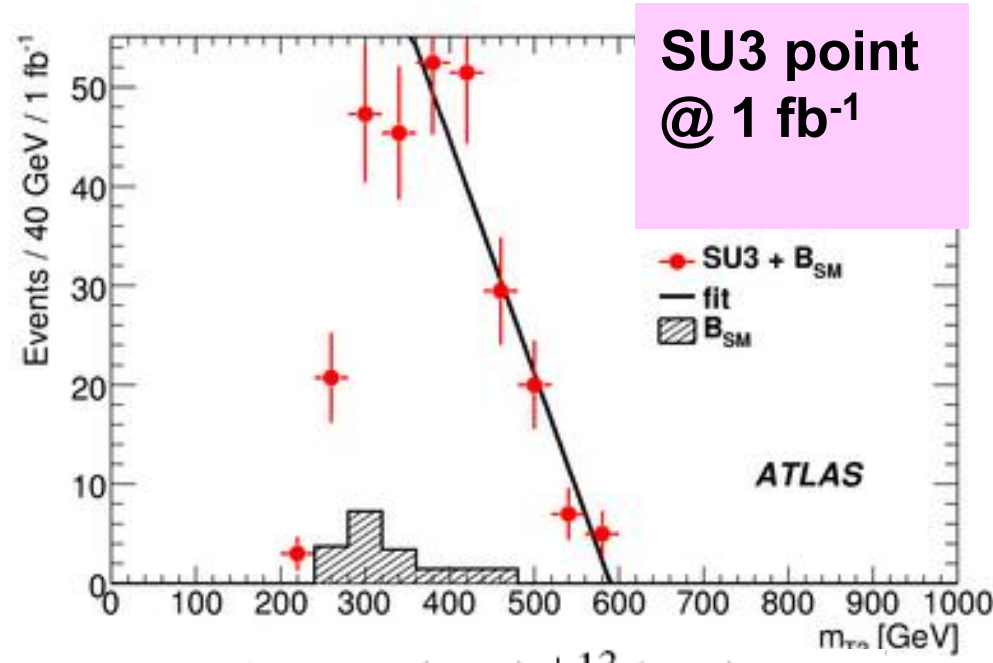

Edge: $590 \pm 9$ (stat) ${ }_{-6}^{+13}$ (sys) $\mathrm{GeV}$ Truth: $611 \mathrm{GeV}$ syst error: choose of fit limits and jet energy scale uncertainty

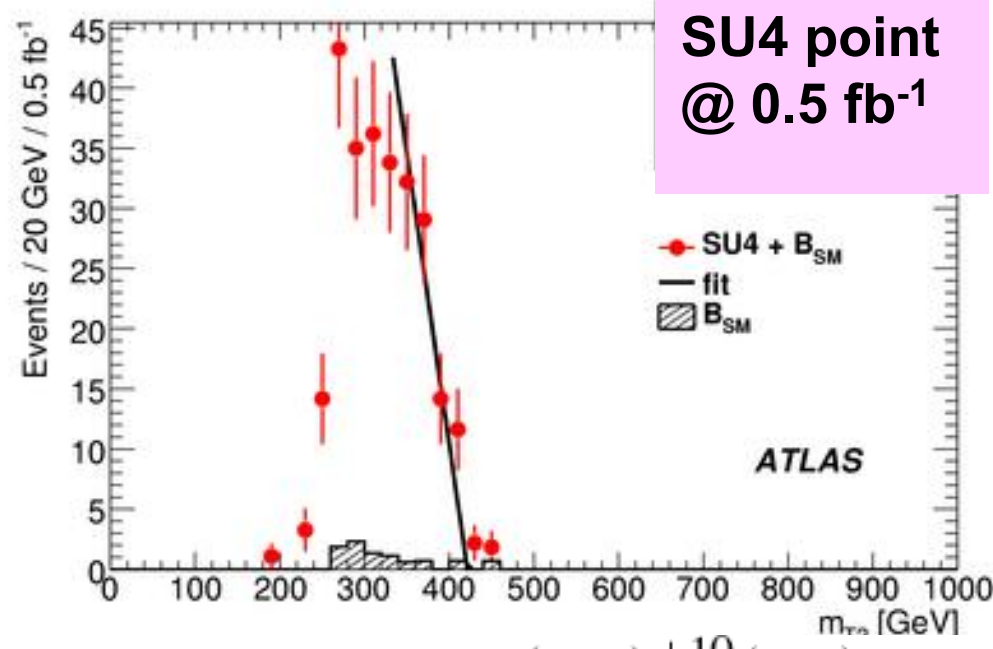

Edge: $421 \pm 17$ (stat) ${ }_{-3}^{+10}$ (sys) $\mathrm{GeV}$ Truth: $406 \mathrm{GeV}$ 


\section{Light Stop Mass}

- In SU4 model the light stop is produced in gluino decay with BR of $42 \%$ :

$$
\tilde{g} \rightarrow \tilde{t}_{1} t \rightarrow \tilde{\chi}_{1}^{ \pm} t b
$$

- The tb invariant mass distribution has an edge at:

$$
M^{\max }(t b)=\left[m_{t}^{2}+\frac{m_{\tilde{t}_{1}}^{2}-m_{\tilde{\chi}_{1}^{ \pm}}^{2}}{2 m_{\tilde{t}_{1}}^{2}}\left(\left(m_{\tilde{g}}^{2}-m_{\tilde{t}_{1}}^{2}-m_{t}^{2}\right)+\sqrt{\left(m_{\tilde{g}}^{2}-\left(m_{\tilde{t}_{1}}-m_{t}\right)^{2}\right)\left(m_{\tilde{g}}^{2}-\left(m_{\tilde{t}_{1}}+m_{t}\right)^{2}\right)}\right)\right]^{1 / 2}
$$

- Other SUSY decays leading to same final state, thus must be suppressed :

$$
\begin{aligned}
& \tilde{g} \rightarrow \tilde{b}_{1} b \rightarrow \tilde{\chi}_{1}^{ \pm} t b, \\
& \tilde{g} \rightarrow \tilde{b}_{1} b \rightarrow \tilde{t}_{1} W b \rightarrow \tilde{\chi}_{1}^{ \pm} b b W \\
& \tilde{g} \rightarrow \tilde{b}_{2} b \rightarrow \tilde{\chi}_{1}^{ \pm} t b, \\
& \tilde{g} \rightarrow \tilde{b}_{2} b \rightarrow \tilde{t}_{1} W b \rightarrow \tilde{\chi}_{1}^{ \pm} b b W
\end{aligned}
$$

- Reconstruct hadronic top only: $t \rightarrow W b \rightarrow q q b$

- The W sideband method is used to estimate SUSY combinatorial background.

- Fit function: triangular smeared with a Gaussian.

syst error: jet energy scale uncertainty and uncertainty on b-tagging efficiency

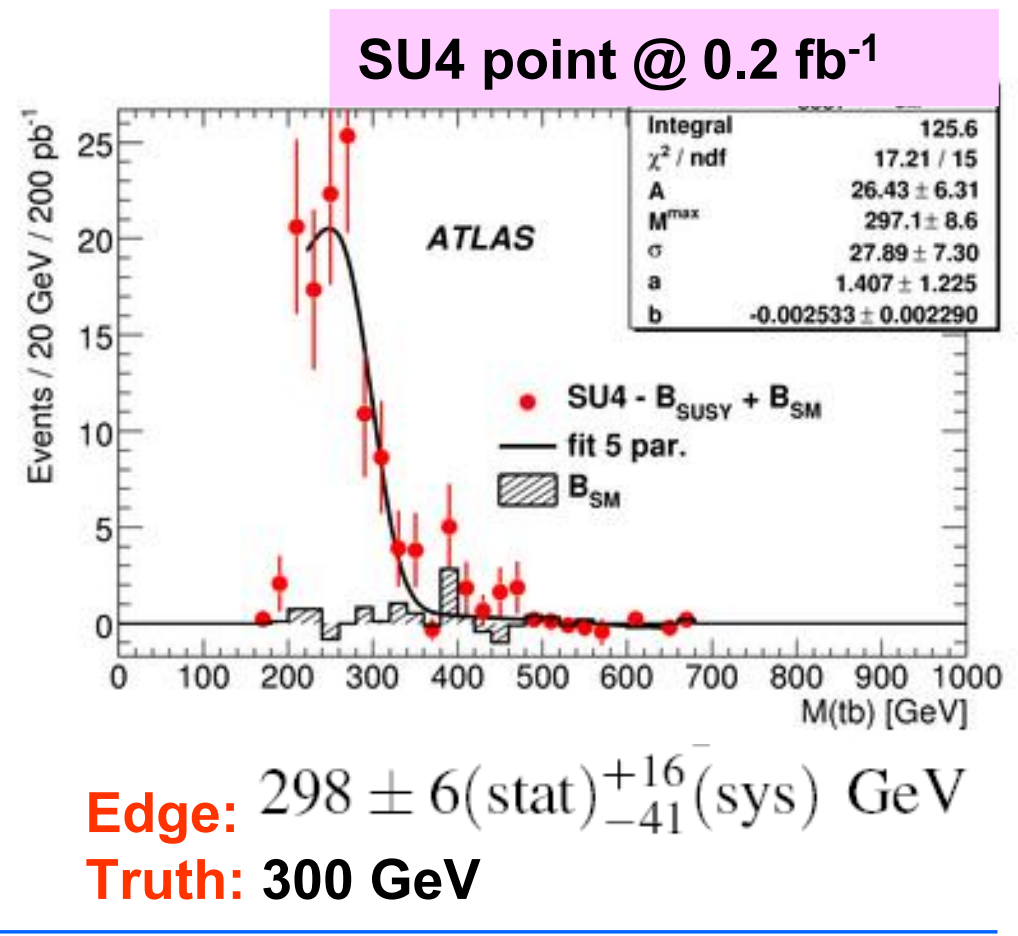




\section{From Endpoints to SUSY Masses}

- Measured endpoints $\rightarrow$ extract involved SUSY particle masses.

- Use a numerical $X^{2}$ minimization based on the MINUIT package:

$$
\chi^{2}=\sum_{k=1}^{n} \frac{\left(m_{k}^{\max }-t_{k}^{\max }\left(m_{\tilde{\chi}_{1}^{0}}, m_{\tilde{\chi}_{2}^{0}}, m_{\tilde{\ell}_{\mathrm{R}}}, m_{\tilde{q}_{\mathrm{L}}}\right)\right)^{2}}{\sigma_{k}^{2}}
$$

SU3 point @ $1 \mathrm{fb}^{-1}$

Measured

Truth

- Use only the endpoints involving leptons and jets $\rightarrow$ five measurements:

$m_{\| l}, m_{l l q}, m_{l l q}$ thres, $m_{l q}^{\text {low }}, m_{l q}^{\text {high }}$

- For SU3, five endpoint measurements for four masses $\rightarrow$ solvable.

- Large statistical error at $1 \mathrm{fb}^{-1}$.

- Mass of LSP is not well determined.

- Mass differences are better measured than absolute masses $\rightarrow$ endpoints most sensitive to mass differences.

\begin{tabular}{l|c|c}
\hline Observable & $\begin{array}{c}\text { SU3 } m_{\text {meas }} \\
{[\mathrm{GeV}]}\end{array}$ & $\begin{array}{c}\text { SU3 } m_{\mathrm{MC}} \\
{[\mathrm{GeV}]}\end{array}$ \\
\hline$m_{\tilde{\chi}_{1}^{0}}$ & $88 \pm 60 \mp 2$ & 118 \\
$m_{\tilde{\chi}_{2}^{0}}$ & $189 \pm 60 \mp 2$ & 219 \\
$m_{\tilde{q}}$ & $614 \pm 91 \pm 11$ & 634 \\
$m_{\tilde{\ell}}$ & $122 \pm 61 \mp 2$ & 155 \\
\hline Observable & $\mathrm{SU} 3 \Delta m_{\text {meas }}$ & $\mathrm{SU} 3 \Delta m_{\mathrm{MC}}$ \\
& {$[\mathrm{GeV}]$} & {$[\mathrm{GeV}]$} \\
\hline$m_{\tilde{\chi}_{2}^{0}-m_{\tilde{\chi}_{1}^{0}}} 100.6 \pm 1.9 \mp 0.0$ & 100.7 \\
$m_{\tilde{q}}-m_{\tilde{\chi}_{1}^{0}}$ & $526 \pm 34 \pm 13$ & 516.0 \\
$m_{\tilde{\ell}}-m_{\tilde{\chi}_{1}^{0}}$ & $34.2 \pm 3.8 \mp 0.1$ & 37.6
\end{tabular}

first error is from MIGRAD, second error is from jet energy scale uncertainty. 


\section{From Endpoints to mSUGRA Parameters}

- Ultimate goal $\rightarrow$ determine SUSY model parameters from endpoint measurements.

- Markov chain analysis $\rightarrow$ obtain a first glimpse of the possible parameter space.

- Use only the endpoints involving leptons and jets plus right-handed squark mass:

$$
m_{\| l}, m_{I l q}, m_{\| l q}^{\text {thres, }} m_{I q}^{\text {low }}, m_{I q}^{\text {high }}, m_{T 2}\left(\tilde{q}_{R}\right)
$$

- Preferred parameters are found around the true parameter points. No further preferrred regions occur. Please see at backup slides.

- Fittino package $\rightarrow$ determine the mSUGRA parameters. Parameter uncertainties and their correlations are obtained from $\mathbf{5 0 0}$ toy fits.

Fit results:

- $\operatorname{sign}(\mu)=+1$ is favored, but $\operatorname{sign}(\mu)=+1$ is not ruled out: $X^{2}=12.6$ versus $X^{2}=15.4$ with $N_{\text {dof }}=11$.

- $M_{0}$ and $M_{1 / 2}$ are well constrained.

- Determination of $A_{0}$ and $\tan \beta$ are more problematic as no information from Higgs sector at low luminosity.

\begin{tabular}{|c|c|c|c|}
\hline \multicolumn{4}{|c|}{ Fit results: SU3 point @ 1 fb-1 } \\
\hline & Truth & Mean & RMS \\
\hline$\overline{\text { Parameter }}$ & SU3 value & fitted value & exp. unc. \\
\hline \multicolumn{4}{|c|}{$\operatorname{sign}(\mu)=+1$} \\
\hline $\tan \beta$ & 6 & 7.4 & 4.6 \\
\hline$M_{0}$ & $100 \mathrm{GeV}$ & $98.5 \mathrm{GeV}$ & $\pm 9.3 \mathrm{GeV}$ \\
\hline$M_{1 / 2}$ & $300 \mathrm{GeV}$ & $317.7 \mathrm{GeV}$ & $\pm 6.9 \mathrm{GeV}$ \\
\hline$A_{0}$ & $-300 \mathrm{GeV}$ & $445 \mathrm{GeV}$ & $\pm 408 \mathrm{GeV}$ \\
\hline \multicolumn{4}{|c|}{$\operatorname{sign}(\mu)=-1$} \\
\hline $\tan \beta$ & & 13.9 & \pm 2.8 \\
\hline$M_{0}$ & & $104 \mathrm{GeV}$ & $\pm 18 \mathrm{GeV}$ \\
\hline$M_{1 / 2}$ & & $309.6 \mathrm{GeV}$ & $\pm 5.9 \mathrm{GeV}$ \\
\hline$A_{0}$ & & $489 \mathrm{GeV}$ & $\pm 189 \mathrm{GeV}$ \\
\hline
\end{tabular}




\section{Conclusions}

- Next step after SUSY discovery (if SUSY exists in nature) will be to select specific SUSY decay chains to measure the properties of new particles.

- We have focused here on exclusive measurements possible with $1 \mathrm{fb}^{-1}$ of data.

- Specific benchmark points in mSUGRA model have been used to demonstrate the precision that can be expected from these measurements, but same/similiar techniques can be applied to much of the SUSY parameter space accessible with the early LHC data.

- With $1 \mathrm{fb}^{-1}$ of data the reconstruction of part of the SUSY mass spectrum will only be possible for favorable SUSY scenarios (low mass).

- Feasibility of SUSY parameter determination has also been demonstrated with $1 \mathrm{fb}^{-1}$ of data.

We are now only few months away from the LHC data! 


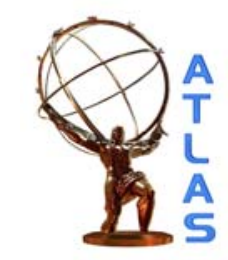

\section{Backup Slides}




\section{SUSY Particle Masses}

\begin{tabular}{|crrrrrrr|}
\hline Particle & SU1 & SU2 & SU3 & SU4 & SU6 & SU8.1 & SU9 \\
\hline$\tilde{d}_{L}$ & 764.90 & 3564.13 & 636.27 & 419.84 & 870.79 & 801.16 & 956.07 \\
$\tilde{u}_{L}$ & 760.42 & 3563.24 & 631.51 & 412.25 & 866.84 & 797.09 & 952.47 \\
$\tilde{b}_{1}$ & 697.90 & 2924.80 & 575.23 & 358.49 & 716.83 & 690.31 & 868.06 \\
$\tilde{t}_{1}$ & 572.96 & 2131.11 & 424.12 & 206.04 & 641.61 & 603.65 & 725.03 \\
$\tilde{d}_{R}$ & 733.53 & 3576.13 & 610.69 & 406.22 & 840.21 & 771.91 & 920.83 \\
$\tilde{u}_{R}$ & 735.41 & 3574.18 & 611.81 & 404.92 & 842.16 & 773.69 & 923.49 \\
$\tilde{b}_{2}$ & 722.87 & 3500.55 & 610.73 & 399.18 & 779.42 & 743.09 & 910.76 \\
$\tilde{t}_{2}$ & 749.46 & 2935.36 & 650.50 & 445.00 & 797.99 & 766.21 & 911.20 \\
\hline$\tilde{e}_{L}$ & 255.13 & 3547.50 & 230.45 & 231.94 & 411.89 & 325.44 & 417.21 \\
$\tilde{v}_{e}$ & 238.31 & 3546.32 & 216.96 & 217.92 & 401.89 & 315.29 & 407.91 \\
$\tilde{\tau}_{1}$ & 146.50 & 3519.62 & 149.99 & 200.50 & 181.31 & 151.90 & 320.22 \\
$\tilde{v}_{\tau}$ & 237.56 & 3532.27 & 216.29 & 215.53 & 358.26 & 296.98 & 401.08 \\
$\tilde{e}_{R}$ & 154.06 & 3547.46 & 155.45 & 212.88 & 351.10 & 253.35 & 340.86 \\
$\tilde{\tau}_{2}$ & 256.98 & 3533.69 & 232.17 & 236.04 & 392.58 & 331.34 & 416.43 \\
\hline$\tilde{g}$ & 832.33 & 856.59 & 717.46 & 413.37 & 894.70 & 856.45 & 999.30 \\
$\tilde{\chi}_{1}^{0}$ & 136.98 & 103.35 & 117.91 & 59.84 & 149.57 & 142.45 & 173.31 \\
$\tilde{\chi}_{2}^{0}$ & 263.64 & 160.37 & 218.60 & 113.48 & 287.97 & 273.95 & 325.39 \\
$\tilde{\chi}_{3}^{0}$ & 466.44 & 179.76 & 463.99 & 308.94 & 477.23 & 463.55 & 520.62 \\
$\tilde{\chi}_{4}^{0}$ & 483.30 & 294.90 & 480.59 & 327.76 & 492.23 & 479.01 & 536.89 \\
$\tilde{\chi}_{1}^{+}$ & 262.06 & 149.42 & 218.33 & 113.22 & 288.29 & 274.30 & 326.00 \\
$\tilde{\chi}_{2}^{+}$ & 483.62 & 286.81 & 480.16 & 326.59 & 492.42 & 479.22 & 536.81 \\
\hline$h^{0}$ & 115.81 & 119.01 & 114.83 & 113.98 & 116.85 & 116.69 & 114.45 \\
$H^{0}$ & 515.99 & 3529.74 & 512.86 & 370.47 & 388.92 & 430.49 & 632.77 \\
$A^{0}$ & 512.39 & 3506.62 & 511.53 & 368.18 & 386.47 & 427.74 & 628.60 \\
$H^{+}$ & 521.90 & 3530.61 & 518.15 & 378.90 & 401.15 & 440.23 & 638.88 \\
$t$ & 175.00 & 175.00 & 175.00 & 175.00 & 175.00 & 175.00 & 175.00 \\
\hline
\end{tabular}




\section{Object/Particle Identification}

- Jets:

- Cone4Tower algorithm with a cone size of 0.4

- Jets matching within a cone of 0.2 an isolated lepton passing the cuts below are discarded (overlap removal)

- Missing ET:

- MET_RefFinal algorithm, calculated from the calorimeter cells, with calibration weights derived separately for cells associated to different objects (jets, electrons, photons, taus, and nonassociated cluster due to the soft part of the event). Source of fake missing energy are not considered here. Require the azimuthal angular separation between the missing ET vector and the three leading jets is larger than 0.2

- Electron:

- EGamma algorithm with "medium" cuts, pT>10GeV, $|\eta|<2.5$

- Reject event if electron is in the crack region $(1.37<|\eta|<1.52)$, calculation of isolation variable is buggy in this region

- Isolated electrons: transverse isolation energy within a cone of 0.2 around the electron is smaller than $10 \mathrm{GeV}$.

- Electron is rejected if it is found within a distance of $0.2<\Delta R<0.4$

- Muon:

- Staco algorithm, $\mathrm{pT}>10 \mathrm{GeV},|\eta|<2.5$, transverse isolation energy within a cone of 0.2 around the muon is smaller than $10 \mathrm{GeV}$

- The track segment match $X^{2}$ is smaller than 100

- Muon is rejected if it is found within a distance of $\Delta R<0.4$ 


\section{Event Selection for Dilepton Inv. Mass}

- In order to select SUSY events and reject the SM background, require the presence of energetic jets and missing energy. The variables used to discriminate SUSY from the SM background are listed in the table below.

- In order to optimize the cuts on these variables, the value of $S$ variable is maximized for each SUSY point. The S variable can be computed from collider data since no Monte Carlo information is used:

$$
S \equiv \frac{N(O S S F)-N(O S O F)}{\sqrt{N(O S S F)+N(O S O F)}}
$$

Best selections for $1 \mathrm{fb}^{-1}$ $\mathrm{m}(\mathrm{II})<\mathrm{m}(\mathrm{II})^{\text {edge }}+10 \mathrm{GeV}$

\begin{tabular}{|l|l|l|l|l|l|l|l|l|l|l|l|l|}
\hline & $\begin{array}{l}\text { pT } \\
\text { (jet1) }\end{array}$ & $\begin{array}{l}\text { pT } \\
\text { (jet2) }\end{array}$ & $\begin{array}{l}\text { pT } \\
\text { (jet3) }\end{array}$ & $\begin{array}{l}\text { pT } \\
\text { (jet4) }\end{array}$ & $\begin{array}{l}\text { Missing } \\
\text { ET }\end{array}$ & $\begin{array}{l}\text { Missing } \\
\text { ET/Meff }\end{array}$ & $\begin{array}{l}\mathrm{S}_{\mathrm{T}} \\
\text { SF } \\
(\text { sig })\end{array}$ & $\begin{array}{l}\text { OF } \\
(\text { sig })\end{array}$ & $\begin{array}{l}\text { SF } \\
\text { (bck) }\end{array}$ & $\begin{array}{l}\text { OF } \\
\text { (bck) }\end{array}$ & S \\
\hline SU3 & 200 & 150 & - & - & 120 & - & - & 120 & 64 & 69 & 53 & 5.1 \\
\hline SU4 & 100 & 50 & - & - & 100 & - & - & 3048 & 1574 & 411 & 419 & 19.9 \\
\hline
\end{tabular}

Compare to "TDR type" cuts: pT(jet1)> $100 \mathrm{GeV}$, pT(jet2-jet4) > $50 \mathrm{GeV}$, MissingET>100 GeV, MissingET/Meff $>0.2, \mathrm{~S}_{\mathrm{T}}>0.2$ 


\section{Markov Chain Likelihood Maps}
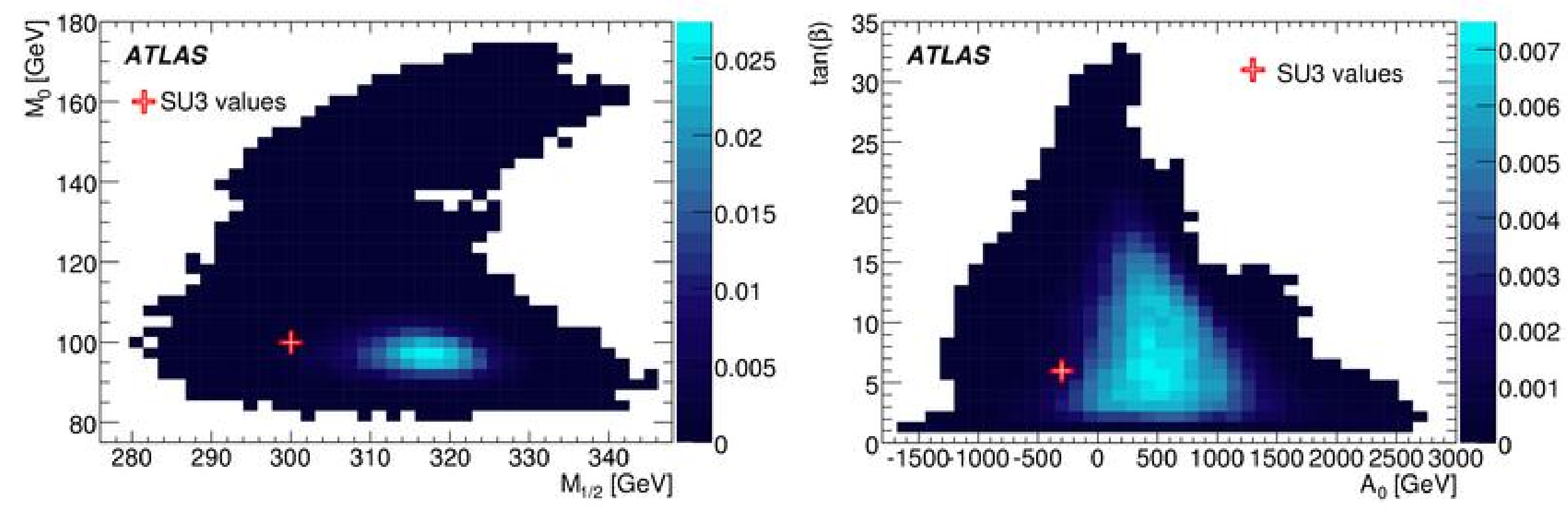